\title{
A Comparison Between Block CEM and Two-Way CEM Algorithms to Cluster a Contingency Table
}

\author{
Mohamed Nadif ${ }^{1}$ and Gérard Govaert ${ }^{2}$ \\ 1 LITA EA3097, Université de Metz, Ile du Saulcy, 57045 Metz, France \\ mohamed.nadif@iut.univ-metz.fr \\ 2 HEUDIASYC, UMR CNRS 6599, Université de Technologie de Compiègne, \\ BP 20529, 60205 Compiègne Cedex, France \\ gerard.govaert@utc.fr
}

\begin{abstract}
When the data consists of a set of objects described by a set of variables, we have recently proposed a new mixture model which takes into account the block clustering problem on the both sets and have developed the block CEM algorithm. In this paper, we embed the block clustering problem of contingency table in the mixture approach. In using a Poisson model and adopting the classification maximum likelihood principle we perform an adapted version of block CEM. We evaluate its performance and compare it to a simple use of CEM applied on the both sets separately. We present detailed experimental results on simulated data and we show the interest of this new algorithm.
\end{abstract}

\section{Introduction}

Cluster analysis is an important tool in a variety of scientific areas such as pattern recognition, information retrieval, micro-array, data mining, and so forth. Although many clustering procedures such as hierarchical clustering, $k$-means or self-organizing maps, aim to construct an optimal partition of objects or, sometimes, of variables, there are other methods, called block clustering methods, which consider simultaneously the two sets and organize the data into homogeneous blocks.

A wide variety of procedures have been proposed for finding patterns in data matrices. These procedures differ in the pattern they seek, the types of data to which they apply, and the assumption on which they rely. Let us mention the works of Hartigan (1975), Bock (1979), Garcia and Proth (1986), Marchotorchino (1987), Govaert (1983, 1995), Arabie and Hubert (1990), Duffy and Quiroz (1991) and Ritschard et al. (2001) who have proposed some algorithms dedicated to different kinds of matrices.

These last years, block clustering (also called biclustering) has become an important challenge in data mining context. In the text mining field, Dhillon (2001) has proposed a spectral block clustering method by exploiting the duality between rows (documents) and columns (words). In the analysis of micro-array 
data where data are often presented as matrices of expression levels of genes under different conditions, block clustering of genes and conditions has permitted to overcome the problem of the choice of similarity on the both sets found in conventional clustering methods (Cheng and Church, 2000). Also, these kinds of methods have practical importance in a wide of variety of applications such as text and market basket data analysis. Typically, the data that arises in these applications is arranged as a two-way contingency or co-occurrence table.

In this paper, we will focus on these kinds of data. The data which we consider is noted $\mathbf{x}$; it is a $r \times s$ data matrix defined by $\mathbf{x}=\left\{\left(x_{i j}\right) ; i \in I, j \in J\right\}$, where $I$ is a categorical variable with $r$ categories and $J$ a categorical variable with $s$ categories. In exploiting the duality between $I$ and $J$, we will study the block clustering problem in embedding it in the mixture approach. We will propose a block mixture model which takes into account the block clustering situation and perform an innovative co-clustering algorithm. This one is based on the alternated application of Classification EM (Celeux and Govaert, 1992) on intermediate data matrices. To propose this algorithm, we set this problem in the classification maximum likelihood (CML) approach (Symons, 1981). This paper deals to compare block CEM and two-way CEM, i.e. CEM applied separately on $I$ and $J$. Results on simulated data are given, confirming that block CEM gives much better performance than two-way CEM.

The paper is organized as follows. In Section 2, we give the necessary background CML approach and we describe the CEM algorithm and its steps when the data is arranged as a two-way contingency. In Section 3, we start by recalling our block mixture model and we describe the block CEM algorithm. In order to compare two-way CEM and block CEM, in Section 4, we perform numerical Monte Carlo simulations. A final section summarizes and indicates the recommended algorithm.

\section{Mixture Model and Clustering}

For convenience, we represent a partition of $I$ into $g$ clusters by $\mathbf{z}=\left(\mathbf{z}_{1}, \ldots, \mathbf{z}_{r}\right)$ where $\mathbf{z}_{i}$, which indicates the component of the row $i$, is represented by $\mathbf{z}_{i}=$ $\left(z_{i 1}, \ldots, z_{i g}\right)$ with $z_{i k}=1$ if row $i$ is in cluster $k$ and 0 otherwise. Then, the $k$ th cluster corresponds to the set of rows $i$ such that $z_{i k}=1$. We will use similar notation for a partition $\mathbf{w}$ into $m$ clusters of the set $J$. In the following, to simplify the notation, the sums and the products relating to rows, columns or clusters will be subscripted respectively by letters $i, j$ or $k$ without indicating the limits of variation, which will be thus implicit. Thus, for example, the sum $\sum_{i}$ stands for $\sum_{i=1}^{r}$ or $\sum_{i, j, k, \ell}$ stands for $\sum_{i=1}^{r} \sum_{j=1}^{s} \sum_{k=1}^{g} \sum_{\ell=1}^{m}$.

\subsection{Approach and the CEM Algorithm}

In the model-based clustering (see for instance (McLachlan and Peel, 2000), it is assumed that the data are generated by a mixture of underlying probability 
distributions, where each component $k$ of the mixture represents a cluster. Thus, the density of the observed data $\mathrm{x}$ is expressed as

$$
f(\mathbf{x} ; \boldsymbol{\theta})=\prod_{i} \sum_{k} \pi_{k} \varphi_{k}\left(\boldsymbol{x}_{i} ; \alpha_{k}\right)
$$

where $\boldsymbol{\theta}=\left(\pi_{1}, \ldots, \pi_{g}, \alpha_{1}, \ldots, \alpha_{g}\right),\left(\pi_{1}, \ldots, \pi_{g}\right)$ are the mixing proportions and $\left(\alpha_{1}, \ldots, \alpha_{g}\right)$ are the parameters of the density components $\varphi_{k}$.

The clustering problem can be studied under mixture model using two different approaches: the maximum likelihood (ML) approach and the classification maximum likelihood (CML) approach (Symons, 1981). In this paper we focus on the second approach.

The ML approach estimates the parameters of the mixture and the partition is derived from these parameters using the maximum a posteriori principle (MAP). In the CML, the partition is added to the parameters to be estimated. The CML approach consists in estimating the parameters of the mixture and the partition. The maximum likelihood estimation of these new parameters leads to optimize in $\boldsymbol{\theta}$ and $\mathbf{z}$ the complete data log-likelihood

$$
L_{C}(\mathbf{z}, \boldsymbol{\theta})=L(\boldsymbol{\theta} ; \mathbf{x}, \mathbf{z})=\log f(\mathbf{x}, \mathbf{z} ; \boldsymbol{\theta})=\sum_{i, k} z_{i k} \log \left(p_{k} \varphi_{k}\left(\boldsymbol{x}_{i} ; \alpha_{k}\right)\right) .
$$

This optimization can be done by the Classification EM (CEM) algorithm (Celeux and Govaert, 1992), a variant of EM (Dempster, Laird and Rubin, 1977), which converts the posterior probabilities $t_{i k}$ 's to a discrete classification in a C-step before performing the M-step.

\subsection{Application to Contingency Table}

In this situation, the contingency table $\mathbf{x}$ is a $r \times s$ data matrix defined by $\mathbf{x}=\left\{\left(x_{i j}\right) ; i \in I, j \in J\right\}$, where $I$ and $J$ are categorical variables with $r$ and $s$ categories. The sum of each row $i$ will be denoted $x_{i}$. Thus, if we note $\boldsymbol{\theta}=$ $\left(\pi_{1}, \ldots, \pi_{g}, \alpha_{11}, \ldots, \alpha_{g s}\right)$ the parameter of the model and $\varphi$ is the multinomial distribution of the $k$-th component, the log-likelihood (up to a constant) can be written as $L(\boldsymbol{\theta} ; \mathbf{x})=\sum_{i} \log \sum_{k} \pi_{k} \alpha_{k 1}^{x_{i 1}} \ldots \alpha_{k s}^{x_{i s}}$, and the complete data $\log$ likelihood as $L(\boldsymbol{\theta} ; \mathbf{x}, \mathbf{z})=\sum_{i, k} z_{i k}\left(\ln \pi_{k}+\sum_{j} x_{i j} \log \alpha_{k j}\right)$.

In clustering context, the use of the mixture model deals to find the component from which each row arises. The CEM algorithm allows us to achieve this goal and the different steps of CEM in this situation are

- E-step: compute the posterior probabilities $t_{i k}^{(c)} \propto \pi_{k} \alpha_{k 1}^{x_{i 1}} \ldots \alpha_{k s}^{x_{i s}}$;

- C-step: the $k$ th cluster of $\mathbf{z}^{(c+1)}$ is defined with $z_{i k}^{(c+1)}=1$ if $k=\operatorname{argmax}_{k=1, \ldots, g} t_{i k}^{(c)}$ and $z_{i k}^{(c+1)}=0$ otherwise;

- M-step: by standard calculations, one arrives at the following re-estimations parameters $\pi_{k}^{(c+1)}=\frac{n_{k}^{(c+1)}}{r}$ and $\alpha_{k j}^{(c+1)}=\frac{x_{k j}}{x_{k}}$ where $n_{k}^{(c+1)}$ is the cardinality of the $k$ th cluster of $\mathbf{z}^{(c+1)}, x_{k j}=\sum_{i} z_{i k}^{(c+1)} x_{i j}$ and $x_{k}$. $=\sum_{j} x_{k j}$. 
Having found the estimate of the parameters and noting $f_{k j}=\frac{x_{k j}}{x}$ where $x_{. .}=\sum_{i, j} x_{i j}$, we can show that, when the proportions are fixed, the maximization of $L(\boldsymbol{\theta} ; \mathbf{x}, \mathbf{z})$ is equivalent to the maximization of the mutual information $I(\mathbf{z}, J)=\sum_{k, j} f_{k j} \log \frac{f_{k j}}{f_{k . f . j}}$ and approximately equivalent to the maximization of the chi-square criterion $\chi^{2}(\mathbf{z}, J)=x_{. .} \sum_{k, j} \frac{\left(f_{k j}-f_{k . f . j}\right)^{2}}{f_{k . f . j}}$. Hence the use of the both criteria $\chi^{2}(\mathbf{z}, J)$ and $I(\mathbf{z}, J)$ supposes implicitly that the data arise from a mixture of multinomial distributions. To tackle the block clustering problem, we can obviously use the CEM on $I$ and $J$ separately (noted 2CEM) but unfortunately it is unaware of the correspondence between $I$ and $J$. It will be seen later that this process is ineffective to detect homogeneous blocs.

\section{Block Mixture Model for Contingency Table}

To study the block clustering problem, we have extended (Govaert and Nadif, 2003) the mixture model to propose a block mixture model defined by the following probability density function

$$
f(\mathbf{x} ; \boldsymbol{\theta})=\sum_{(\mathbf{z}, \mathbf{w}) \in \mathcal{Z} \times \mathcal{W}} \prod_{i} \pi_{z_{i}} \prod_{j} \rho_{w_{j}} \prod_{i, j} \varphi\left(x_{i j} ; \boldsymbol{\alpha}_{z_{i} w_{j}}\right)
$$

where $\boldsymbol{\theta}=\left(\boldsymbol{\pi}, \boldsymbol{\rho}, \boldsymbol{\alpha}_{11}, \ldots, \boldsymbol{\alpha}_{g m}\right), \boldsymbol{\pi}=\left(\pi_{1}, \ldots, \pi_{g}\right)$ and $\boldsymbol{\rho}=\left(\rho_{1}, \ldots, \rho_{m}\right)$ are the mixing proportions and $\varphi\left(x, \boldsymbol{\alpha}_{k \ell}\right)$ is a probability density function defined on the real set $\mathbb{R}$.

Counts in the $r \times s$ cells of a contingency table are typically modelled as random variables. In our situation, we assume that for each block $k \ell$ the values $x_{i j}$ are distributed according the Poisson distribution $\mathcal{P}\left(\alpha_{i} \beta_{i} \delta_{k \ell}\right)$ for which the probability mass function is

$$
\frac{e^{-\alpha_{i} \beta_{j} \delta_{k \ell}}\left(\alpha_{i} \beta_{j} \delta_{k \ell}\right)^{x_{i j}}}{x_{i j} !} .
$$

The Poisson parameter is split into $\alpha_{i}$ and $\beta_{j}$ the effects of the row $i$ and the column $j$ and $\delta_{k \ell}$ the effect of the block $k \ell$. Because the aim is to maximize the complete data log-likelihood not only depending on $\boldsymbol{\theta}$ but on $\mathbf{z}, \mathbf{w}$, an adapted re-parametrization of the Poisson distribution becomes necessary. To this end, we impose some constraints and we assume that $\sum_{\ell} \beta_{\ell} \delta_{k \ell}=1$ and $\sum_{k} \alpha_{k} \delta_{k \ell}=1$ with $\alpha_{k}=\sum_{i, k} z_{i k} \alpha_{i} \beta_{\ell}=\sum_{j, \ell} w_{j \ell} \beta_{j}$.

To tackle the simultaneous partitioning problem, we will use the CML approach, which aims to maximize the classification log-likelihood called complete data log-likelihood associated to the block mixture model. With our model, the complete data are $(\mathbf{z}, \mathbf{w}, \mathbf{x})$ and the classification log-likelihood is given by

$$
L_{c}(\mathbf{z}, \mathbf{w}, \boldsymbol{\theta})=L(\boldsymbol{\theta} ; \mathbf{x}, \mathbf{z}, \mathbf{w})=\log (p(\mathbf{z} ; \boldsymbol{\theta}) p(\mathbf{w} ; \boldsymbol{\theta}) f(\mathbf{x} \mid \mathbf{z}, \mathbf{w} ; \boldsymbol{\theta})) .
$$

To maximize $L_{c}(\mathbf{z}, \mathbf{w}, \boldsymbol{\theta})$, like in Govaert and Nadif (2003) we propose to maximize alternatively the classification log-likelihood with $\mathbf{w}$ and $\boldsymbol{\rho}$ fixed and 
then with $\mathbf{z}$ and $\boldsymbol{\pi}$ fixed. By noting $x_{i \ell}=\sum_{j} w_{j \ell} x_{i j}$, the classification $\log$ likelihood can be written as

$$
L_{c}(\mathbf{z}, \mathbf{w}, \boldsymbol{\theta})=\sum_{i, k} z_{i k} \log \pi_{k}+\sum_{j, \ell} w_{j \ell} \log \rho_{\ell}+\sum_{i, k} z_{i k} \sum_{\ell} x_{i \ell} \log \delta_{k \ell} .
$$

If we note $\mathbf{u}_{i}=\left(x_{i 1}, \ldots, x_{i \ell}, \ldots, x_{i m}\right)$ and $\gamma_{k \ell}=x_{. \ell} \delta_{k \ell}$, the classification log-likelihood can be decomposed into two terms

$$
L_{c}(\mathbf{z}, \mathbf{w}, \boldsymbol{\theta})=L_{c}(\mathbf{z}, \boldsymbol{\theta} / \mathbf{w})+g(\mathbf{x}, \mathbf{w}, \boldsymbol{\rho})
$$

where the first one, can be written as

$$
L_{c}(\mathbf{z}, \boldsymbol{\theta} / \mathbf{w})=\sum_{i, k} z_{i k} \log \left(\pi_{k} \Phi\left(\mathbf{u}_{i}, \gamma_{k}\right)\right)
$$

where $\Phi\left(\mathbf{u}_{i}, \gamma_{k}\right)$ is the multinomial distribution for $x_{i 1}, \ldots, x_{i m}$ with the probabilities $\gamma_{k 1}, \ldots, \gamma_{k m}$ and the second one can be written as

$$
g(\mathbf{x}, \mathbf{w}, \boldsymbol{\rho})=\sum_{j, \ell} w_{j \ell} \log \rho_{\ell}-\sum_{\ell} x_{. \ell} \log x_{. \ell} .
$$

Hence, $L_{c}(\mathbf{z}, \boldsymbol{\theta} / \mathbf{w})$, called in the followings conditional classification loglikelihood, corresponds to the complete log-likelihood associated to a classical mixture model defined on the samples $\mathbf{u}_{1}, \ldots, \mathbf{u}_{r}$. As $g(\mathbf{x}, \mathbf{w}, \boldsymbol{\rho})$ does not depend on $\mathbf{z}$, maximizing $L_{c}(\mathbf{z}, \mathbf{w}, \boldsymbol{\theta})$ for $\mathbf{w}$ fixed is equivalent to maximize the conditional classification $\log$-likelihood $L_{c}(\mathbf{z}, \boldsymbol{\theta} / \mathbf{w})$, which can be done by the CEM algorithm applied to the multinomial mixture model. The different steps of CEM are

- E-step: compute the posterior probabilities $t_{i k}^{(c)}$;

- C-step: the $k$ th cluster of $\mathbf{z}^{(c+1)}$ is defined with $z_{i k}^{(c+1)}=1$ if $k=\operatorname{argmax}_{k=1, \ldots, g} t_{i k}^{(c)}$ and $z_{i k}^{(c+1)}=0$ otherwise.

- M-step: by standard calculations, one arrives at the following re-estimations parameters

$$
\pi_{k}^{(c+1)}=\frac{\# z_{k}^{(c+1)}}{r} \quad \text { and } \quad \delta_{k \ell}^{(c+1)}=\frac{x_{k \ell}}{x_{k \cdot x_{\ell}}}
$$

where \# denotes the cardinality and

$$
x_{k \ell}=\sum_{i} z_{i k}^{(c+1)} x_{i \ell}=\sum_{i j} z_{i k}^{(c+1)} w_{j \ell} x_{i j} .
$$

In the same way, we can show that

$$
L_{c}(\mathbf{z}, \mathbf{w}, \boldsymbol{\theta})=L_{c}(\mathbf{w}, \boldsymbol{\theta} / \mathbf{z})+g(\mathbf{x}, \mathbf{z}, \boldsymbol{\pi})
$$

where

$$
g(\mathbf{x}, \mathbf{z}, \boldsymbol{\pi})=\sum_{i, k} z_{i k} \log \pi_{k}-\sum_{k} x_{k} \log x_{k} .
$$


does not depend on $\mathbf{w}$ and $L_{c}(\mathbf{w}, \boldsymbol{\theta} / \mathbf{z})$ corresponds to the complete log-likelihood associated to a classical mixture model defined on the samples $\mathbf{v}_{1}, \ldots, \mathbf{v}_{s}$ where $\mathbf{v}_{j}=\left(x_{1 j}, \ldots, x_{k j}, \ldots, x_{g j}\right)$ with $x_{k j}=\sum_{i} z_{i k} x_{i j}$ and therefore develop the different steps of the CEM algorithm applied on $\mathbf{v}_{1}, \ldots, v_{s}$ to maximize $L_{c}(\mathbf{z}, \mathbf{w}, \boldsymbol{\theta})$ for $\mathbf{z}$ fixed.

Finally, we can describe easily the different steps of the algorithm called block CEM and noted BCEM:

1. Start from an initial position $\left(\mathbf{z}^{(0)}, \mathbf{w}^{(0)}, \boldsymbol{\theta}^{(0)}\right)$.

2. Computation of $\left(\mathbf{z}^{(c+1)}, \mathbf{w}^{(c+1)}, \boldsymbol{\theta}^{(c+1)}\right)$ starting from $\left(\mathbf{z}^{(c)}, \mathbf{w}^{(c)}, \boldsymbol{\theta}^{(c)}\right)$ :

(a) Computation of $\mathbf{z}^{(c+1)}, \boldsymbol{\pi}^{(c+1)}, \delta^{\left(c+\frac{1}{2}\right)}$ using the CEM algorithm on the data $\left(\mathbf{u}_{1}, \ldots, \mathbf{u}_{r}\right)$ starting from $\mathbf{z}^{(c)}, \boldsymbol{\pi}^{(c)}, \delta^{(c)}$.

(b) Computation of $\mathbf{w}^{(c+1)}, \boldsymbol{\rho}^{(c+1)}, \delta^{(c+1)}$ using the CEM algorithm on the data $\left(\mathbf{v}_{1}, \ldots, \mathbf{v}_{s}\right)$ starting from $\mathbf{w}^{(c)}, \boldsymbol{\rho}^{(c)}, \delta^{\left(c+\frac{1}{2}\right)}$.

3. Iterate the steps 2 until the convergence.

\section{Numerical Experiments}

To illustrate the behavior of our algorithms BCEM and 2CEM, we studied their performances on simulated data. We selected twenty five kinds of data arising from $3 \times 2$-component Poisson block mixture in considering firstly the situation where the proportions are equal proportions $\left(\pi_{1}=\pi_{2}=\pi_{3}\right.$ and $\left.\rho_{1}=\rho_{2}\right)$. These data are obtained by varying the following parameters: the degree of overlapping which depends on the parameters $\boldsymbol{\theta}=(\boldsymbol{\pi}, \boldsymbol{\rho}, \delta)$, and the sizes $r$ and $s$. This degree of overlapping can be measured by the Bayes error corresponding to our model. Its computation being theoretically difficult, we used Monte Carlo simulations and evaluated this error by comparing the simulated partitions and those we obtained by applying a C-step. Five degrees of overlapping have been considered and are approximatively equal to $6 \%, 11 \%, 16 \%, 18 \%, 20 \%$. Concerning the size, we took $r \times s=(30 \times 100),(50 \times 100),(100 \times 100),(500 \times 100)$ and $(1000 \times 100)$.

For each of these 25 data structures, we generated 30 samples and for each sample, we ran BCEM and CEM 100 times starting from random situations and selected the best solution for each method. In order to summarize the behavior of these algorithms, we used the proportion of misclassified points "error rate" occurring for each sample.

The results obtained are displayed in Table 1. For each data set and each algorithm, we summarize the 30 trials with the means and standard deviations of error rates obtained by comparing the partitions obtained by the both methods and the simulated partitions. In Table 2, we report the means and standard deviations of running times.

From these experiments, the main point arising are the following.

- The version 2CEM working on the two sets separately is suitably effective only when the clusters are well separated. This shows the risk of the use of such methods when the clusters are ill-separated. 
Table 1. Comparison of BCEM and 2CEM for 30 kinds of data : means and standard deviations of error rates

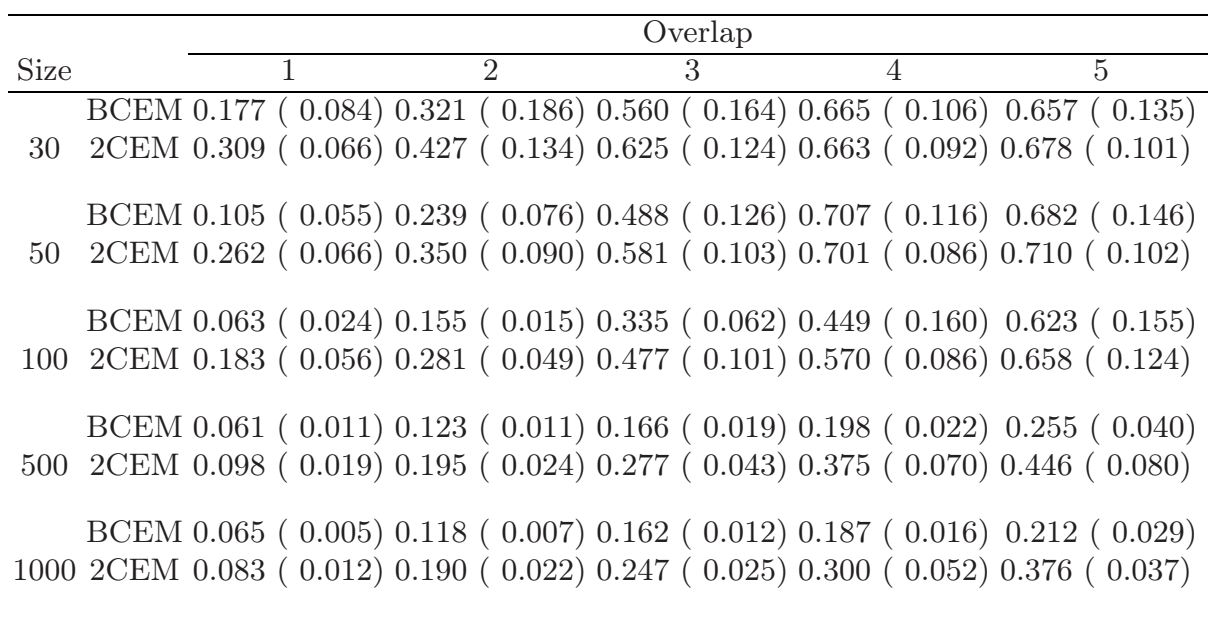

Table 2. Comparison of BCEM and 2CEM for 30 kinds of data : means and standard deviations of running times

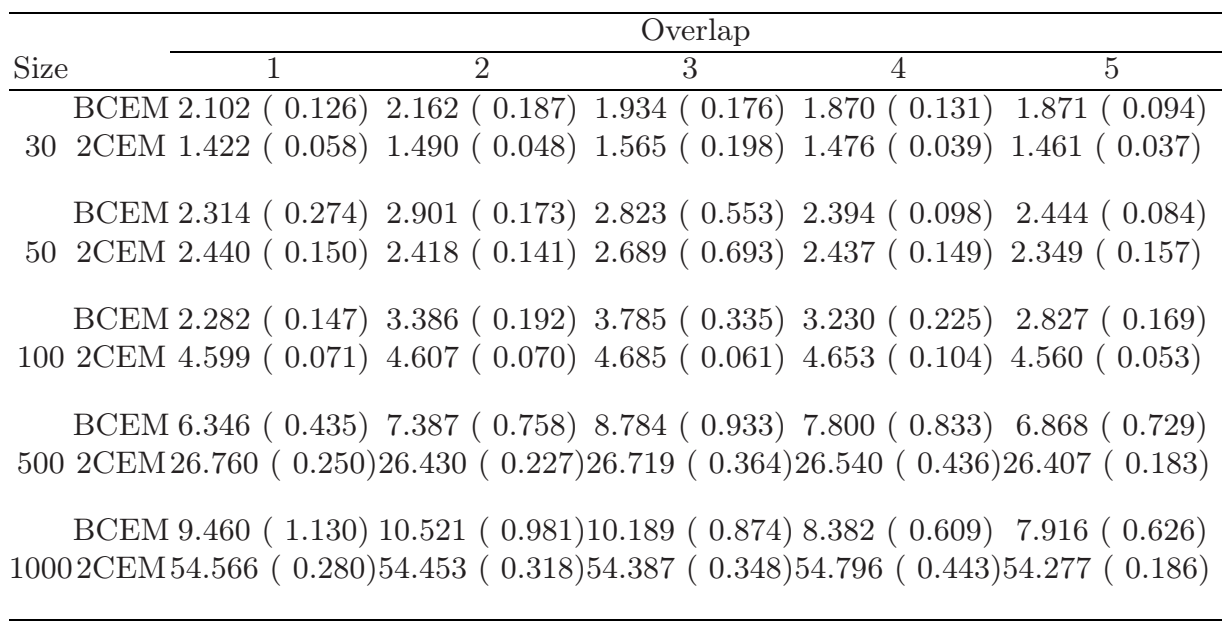

- Incontestably BCEM outperforms 2CEM. The results are very encouraging and its performance increases with the size of data.

- It appears clearly that BCEM is undoubtedly faster as soon as the size is large enough.

We carried out other simulations on large data sets with proportions dramatically different, not included in this text, which confirms these remarks. 


\section{Conclusion}

Setting the problem of block clustering under the CML approach, we have compared block CEM and two-way CEM. The first one gives encouraging results on simulated data and real data and is therefore strongly recommended : it is faster and better than two-way CEM. Currently, we are evaluating block CEM on other large real data sets. In this paper, we have considered the block clustering for contingency tables under the CML approach and, as in Govaert and Nadif (2005a, 2005b) for binary data, it would be interesting to study the block clustering of contingency table under the ML and fuzzy approaches.

\section{References}

Arabie, P., J., H.L.: The bond energy algorithm revisited. IEEE Transactions on Systems, Man, and Cybernetics 20 (1990) 268-274

Bock, H.: Simultaneous clustering of objects and variables. In Diday, E., ed.: Analyse des Données et Informatique, INRIA (1979) 187-203

Celeux, G., Govaert, G.: A classification em algorithm for clustering and two stochastic versions. Computational Statistics and Data Analysis 14 (1992) 315-332

Cheng, Y., Church, G.: Biclustering of expression data. In: Proceedings of the Eighth International Conference on Intelligent Systems for Molecular Biology (ISMB). (2000) 93-103

Dempster, A.P., Laird, N.M., Rubin, D.B.: Maximum likelihood from incomplete data via the em algorithm (with discussion). Journal of the Royal Statistical Society B 39 (1977) 1-38

Dhillon, I.: Co-clustering documents and words using bipartite spectral graph partioning. In: ACM SIGKDD Conference, San Francisco, USA. (2001) 269-274

Duffy, D.E., Quiroz, A.J.: A permutation-based algorithm for block clustering. Journal of Classification 8 (1991) 65-91

Garcia, H., Proth, J.M.: A new cross-decomposition algorithm: The GPM comparison with the bond energy method. Control and Cybernetics 15 (1986) 155-165

Govaert, G.: Classification croisée. Thèse d'état, Université Paris 6, France (1983)

Govaert, G.: Simultaneous clustering of rows and columns. Control and Cybernetics 24 (1995) 437-458

Govaert, G., Nadif, M.: Clustering with block mixture models. Pattern Recognition 36 (2003) 463-473

Govaert, G., Nadif, M.: An EM algorithm for the block mixture model. IEEE Transactions on Pattern Analysis and Machine Intelligence 27 (2005) 643-647

Govaert, G., Nadif, M.: Fuzzy clustering to estimate the parameters of block mixture models. Soft Computing (in press, 2005)

Hartigan, J.A.: Clustering Algorithms. Wiley, New York (1975)

Marchotorchino, F.: Block seriation problems: A unified approach. Applied Stochastic Models and Data Analysis 3 (1987) 73-91

McLachlan, G.J., Peel, D.: Finite Mixture Models. Wiley, New York (2000)

Ritschard, G. Zighed, D., Nicoloyannis, N., Maximisation de l'association par regroupement de lignes ou de colonnes d'un tableau croisé. Revue de Mathématiques \& Sciences Humaines 39 (2001) 81-97

Symons, M.J.: Clustering criteria and multivariate normal mixture. Biometrics 37 (1981) 35-43 